\title{
Specialised Nursing-based Transitional Care in Elderly Patients with Chronic Diseases
}

\author{
Xia Lyu ${ }^{1 *}$, Jinling $\mathrm{Li}^{1}$, Wuhong Deng ${ }^{1}$, Hongying $\mathrm{Hu}^{1}$, Jie Zeng ${ }^{1}$ and Jenny Song ${ }^{2}$ \\ ${ }^{1}$ Shenzhen People's Hospital, Shenzhen, China \\ ${ }^{2}$ Waikato Institute of Technology, Hamilton, New Zealand
}

"Corresponding author: Xia Lyu, Associate Director of Nursing Department, Associate Director of Continuing Care Department, Registered Nurse, Shenzhen People's Hospital, Shenzhen, China, Tel: 0755-22942821; E-mail: Ivxia16@163.com

Received date: January 08, 2018; Accepted date: January 30, 2018; Published date: February 07, 2018

Copyright: (c) 2018 Lyu X, et al. This is an open-access article distributed under the terms of the Creative Commons Attribution License, which permits unrestricted use, distribution, and reproduction in any medium, provided the original author and source are credited

\begin{abstract}
The purpose of this study is to explore the influence of transitional care by specialty nurses (SN) for elderly patients with dementia.

A total of 104 patients with dementia suffering from various diseases of the elderly were invited to participate in this research project in the Department of Internal Medicine in Shenzhen People's hospital from September 2015 to February 2016. Using purposive sampling, the patients were assigned into two groups: the control group and the observation group. Patients in both groups were evaluated before discharge. Patients in the control group were given only routine nursing care and telephone follow-up in the first month after discharge. Patients in the observation group were given transitional care on the top of the usual care offered. The unscheduled readmission rate, compliance behavior and incidence of complications for those discharged with tubes after discharge were compared between the groups.
\end{abstract}

The results showed that the unscheduled readmission rate of patients in the observation group decreased, compliance behavior improved and the incidence of complications for those discharged with indwelling catheters (IDC) was reduced. There were statistically significant differences in data between the groups.

Transitional care led by specialized nurses can lower the readmission rate, reduce complications, and improve compliance behavior, which results in a quick recovery for patients after discharge.

Keywords: Specialty nurses; Transitional care; Patients with dementia

\section{Introduction}

By the end of 2012, there were 194 million people aged 60 years and over in China, accounting for $14.3 \%$ of the total population. Among them, 22.73 million people were aged 80 years and above, of whom 36 million were dependent on carers for their needs; an old-age dependency ratio of $20.66 \%$ [1]. Chronic diseases mainly refer to a group of diseases represented by cardiovascular and cerebrovascular diseases, diabetes mellitus, malignancy, chronic obstructive pulmonary diseases, and similar ailments. The normal practice in China has been that the hospital staff discontinues their relationship with the patient upon discharge. Traditional medical care services have mainly focused on the treatment and care of patients during their hospitalization [2]. There has been no provision in China for continued care after discharge. It has been a challenge to meet patients' care needs after discharge. Buurman et al. [3] pointed out that $30 \%$ of patients had a significant decline in daily living activity within 3 months after discharge, especially among the elderly patients. One of the key reasons for this decline has been due to the lack of continuing monitoring of the patients after their transition from hospital to home [4].
Attention to the patient's transitional care has become a new nursing focus extending care beyond the hospital and into the family and community in response to health problems and health needs in patients during the transition period. The American Geriatric Society defines transitional care as the design of a series of care services to ensure that the health care services received by patients have been coordinated in order to provide continuity of care when the patient moves between different health care amenities or transfers between different levels of health care institutions. Transitional care is also aimed at preventing and reducing the deterioration of health for highrisk patients [5].

With the growing number of elderly in China, chronic disease management and long-term care needs are likewise increasing. For this reason, the management of the Shenzhen People's Hospital has begun to look at the professional development of nurses to encourage them to further their studies to become advanced nursing practitioners. Specialty nurses are those who have completed specialty nursing educational courses and have acquired the appropriate qualifications [6,7]. The Shenzhen People's Hospital established a Specialized Nursing-based Transitional Care Services Department in 2015 to carry out transitional services for elderly patients after discharge. 


\section{Research Methods}

\section{Research subjects}

A total of 104 elderly patients with chronic diseases hospitalized in the internal medicine departments in our hospital from February 2016 to February 2017 were invited to participate in the research. Of this number, there were 14 cases of emphysema, 25 cases of coronary heart disease and 65 cases of stroke. In addition, there were 56 patients with in-dwelling catheters and 35 patients with in-dwelling gastric tubes. The patients were then divided into an observation group and a control group according to random selection, with 52 cases in each group. There was no significant difference in gender, age, disease condition and clinical manifestations between the two groups $(\mathrm{P}>0.05)$. The inclusion criteria: (1) age $\geq 65$ years; (2) chronic disease hospital stay $\geq$ 1 week; (3) accompanied by varying degrees of functional defects and weakness; (4) patients who volunteered to participate in the research and complete the evaluation process. The main criteria for exclusion were: (1) the patient had mental illness or was cognitively dysfunctional (2) they suffered an advanced tumor or (3) had chronic diseases that could be exacerbated over this time.

Ethical approval was granted by the Hospital Research Ethics Committee. All patients provided written informed consent before participating in the study. Routine interventions were adopted in the control group. Before discharge, the nurse responsible for the patient would provide them and the members of their family with health education explaining the nature of the disease, what medication was required, what the patient should eat, an appropriate exercise regime and follow-up information. Within one month after discharge, patients would be followed up to fill out a follow-up record sheet by phone, with a further call to have an opportunity to have their questions answered. As well as these phone conversations, patients could visit the community or hospital care clinic. For the intervention group, the Transitional Care Service Department would arrange for an SN to visit the patient's home for their transitional care services.

\section{Transitional care service team}

The hospital set up a Transitional Care Service Department under the Nursing Department composed of five nursing service groups, i.e., those working in geriatric care, orthopedics, neurology, endocrine and spinal surgery. Each group was led by a SN and the group members were composed of a SN and other senior nurses who had more than five years of clinical experience. The group leader coordinated the work of each group.

\section{Transitional care services}

- One week before discharge, the transitional care service team would conduct pre-discharge care evaluations developing care measures and plan according to the patients' existing problems. A Transitional Care Service archive also needed to be established detailing the name, gender, age, diagnosis, nursing problems, family condition, home address and contact telephone number.

- One week after discharge, patients would be followed up by a group leader and a group member who would respond to any issues arising and provide some additional educational advice. They would also evaluate the patients' self-care ability and discuss potential problems such as falls, pressure sores, pain scales and give further guidance.
- Patients with pressure sores or incontinence associated dermatitis should be followed up by wound SN continuously, who would carry out a home evaluation, dressing the patient, providing basic care guidance and dietary advice.

- During this home evaluation, the SN would also provide the patients with indwelling tubes with guidance for their care and maintenance.

- Patients would be followed up by phone within one month after discharge to fill out the telephone follow-up record sheet. This follow-up would also provide an opportunity for patient medication and rehabilitation training advice. It would allow the team to find out the psychological state of the patients or their family with an offer of further support and comfort.

- A home visit would be conducted for patients at the end of the first, second and third month after the patient's discharge, to attend to any problems that arose from the previous home visit and telephone follow-up and to assist with any further concerns that might have arisen.

- A follow-up portfolio would be established for each patient to detail the patient's progress: their treatment and discharge situation, their medication and the conditions recorded through each visit and telephone follow-up. If there were any wounds and/or tubes, the treatment process would be recorded after each visit, attached with pictures.

- The follow-up patients' portfolio would be submitted to the Transitional Care Service Department for unified management.

\section{Evaluation method}

The unscheduled readmission rate, compliance rate, and incidence of complications with tubes after discharge were compared between the two groups within three months.

Unscheduled readmission rate=Number of patients of unscheduled readmission/Number of patients in the group $\times 100 \%$

Evaluation on patient's compliance behavior according to the designed compliance behavior, including full compliance of five items (diet, activities, medication, examination, emotion, patient's adherence), partial compliance ( 2 to 4 items), and non-compliance (0 to 1 item $)$. Compliance rate $=[($ full compliance/partial compliance $) /$ number of patients in this group] $\times 100 \%$

Various types of tube congestion, bleeding, exudate, infection were included as complications with tube in place. The incidence of complications with tube in place after discharge $=$ The number of patients with complications with tube in place/number of patients with tube in place in the group $\times 100 \%$

\section{Statistical analysis}

Data processing was performed by SPSS19.0 statistical software. Measurement data were expressed by $\chi \pm S D$; corrected $\chi^{2}$ test was performed for enumeration data; and rank sum test was performed for ranked data.

\section{Results}

Clinical data on the patients with senile chronic diseases receiving routine nursing care and transitional nursing (Table 1). 


\begin{tabular}{|c|c|c|c|c|}
\hline & Control group & Observation group & $x^{2}$ & $P$ value \\
\hline Sex & & & 0.17 & 0.684 \\
\hline Male & 18 & 20 & & \\
\hline Female & 34 & 32 & & \\
\hline Age & $0.52 \pm 7.20$ & $70.82 \pm 7.14$ & & 0.832 \\
\hline Diagnosis & & & 1.632 & 0.442 \\
\hline Chronic obstructive pulmonary disease & 6 & 8 & & \\
\hline Coronary heart disease & 15 & 10 & & \\
\hline Cerebral apoplexy & 30 & 35 & & \\
\hline Number of patients admitted & & & 3.983 & 0.046 \\
\hline Scheduled & 44 & 50 & & \\
\hline Unschedule & 8 & 2 & & \\
\hline Compliance behavior & & & 7.792 & 0.005 \\
\hline Compliance & 37 & 48 & & \\
\hline Non-compliance & 15 & 4 & & \\
\hline Complications with tube in place after discharge & & & 4.727 & 0.03 \\
\hline Yes & 12 & 4 & & \\
\hline No & 40 & 48 & & \\
\hline
\end{tabular}

Table 1: Clinical data on the patients with senile chronic diseases receiving routine nursing care and transitional nursing.

\section{Discussion}

The transitional services are supported by national policies. In 2016, the National Health and Family Planning Commission formulated the China Nursing Care Development Plan (2016-2020), which pointed out that services in the nursing field should be expanded (1) to carry out transitional care services (2) to encourage medical institutions to give full play to professional skills and talents (3) to provide various kinds of transitional care services for discharged patients (4) to extend the care services to the family in community and (5) to gradually improve the services content and means, thus guaranteeing the continuity of care services [8]. The Plan showed that these transitional care services would be a direction and focus of the development of Chinese care services.

With the rapid development of medical care, such services should not be limited to simple technical operations, but involve the development of specialist technical practices based on better levels of predictability of needed patient care. The SN-based Transitional Care Services would not only provide patients with standardized and comprehensive guidance technically, but also predict to determine the patient's disease change, so as to ensure the implementation of transitional services and quality of services.

Elderly patients have complex diseases and suffer more complications as a result. Being discharged from a hospital setting does not mean that complete rehabilitation has occurred. The condition of the patient could change at any time after the discharge, with slow recovery after acute illness. By providing continuous follow-up care, however, self-care through greater health awareness was anticipated. In this study, the Transitional Care Service Department conducted a comprehensive assessment of the patient before discharge to identify any potential nursing problems and develop a personalized transitional care program. As well as tracking the patient's compliance behavior, instances of complications with tubes were noted through both a telephone follow-up and family visit. On the basis of the result of the patient's progress, timely and appropriate measures were taken to prevent the patient deteriorating again. The family visits, site observations of the patient's home, provided valuable information for nurses about the patient's living environment and day-to-day lifestyle, allowing them to take corrective measures through health education. Study results showed that, in the observation group, the unscheduled readmission rate within three months decreased significantly. While the compliance behavior had improved the incidence of complications with tubes in place decreased. The difference between the two groups was statistically significant.

China has entered an aging society which produces a series of problems, most of which is medical care for the aged. According to the recent medical reforms, diagnosis and treatment is improving. In a similar vein, the Transitional Care Services can support the needs of local health services by promoting patient rehabilitation, reducing the incidence of complications, reducing the incidence of rehospitalization and the keeping down the costs of the health services overall. 
Citation: Lyu X, Li J, Deng W, Hu H, Zeng J, et al. (2018) Specialised Nursing-based Transitional Care in Elderly Patients with Chronic Diseases.

\section{Conclusion}

Transitional Services are a relatively new feature of the health care landscape in China. No unified standard has been enacted for the process, object, content and time component of these services. It is still in a stage of continuous development and improvement. Tribute must be paid to the SNs' professional expertise in improving the various standards of the Transitional Care Services in guaranteeing the quality of treatment and enhancing the satisfaction of the patient.

\section{References}

1. Wu YS (2013) China aging development report. Beijing: Social Sciences Academic Press

2. Liu MJ, Ye Y, Zhang FL (2013) Analysis on basic elements of the definition of transitional care based on content analysis method. J Nurs Sci 28: 74-77.
3. Buurman BM, Parlevliet JL, Deelen BA (2010) A randomised clinical trial on a comprehensive geriatric assessment and intensive home follow-up after hospital discharge: The transitional care bridge. BMC Health Serv Res 10: 296.

4. Piraino E, Heckman G, Glenny C (2012) Transitional care programs: Who is left behind? A systematic review. Int J Integr Care 12: 132.

5. Shortell SM, Rundall TG, Hsu J (2007) Improving patient care by linking evidence-based medicine and evidence-based management. JAMA 9: 673-676.

6. Guo YH (2004) On senior nurses development status and trends. Chin Nurs Manag 4: 19-20.

7. Hu M, Liu YL, Zhang YX (2010) Effect of transitional care on the quality of life of patients with myocardial infarction after discharge. Chin J Modern Nurs 16: 3262-3264.

8. Ministry of Health (2016) Nursing career development plan (2016-2020). China Nursing Management. 